\title{
ANALYSIS AND COMPUTER TOOLS FOR SEPARATION PROCESSES INVOLVING NONIDEAL MIXTURES
}

\author{
Progress Report
}

for Period December 1, 1989 - November 30, 1992

Angelo Lucia

Clarkson University

Potsdam, NY 13699-5705

May 1992

\section{DISCLAIMER}

\begin{abstract}
This report was prepared as an account of work sponsored by an agency of the United States Government. Neither the United States Government nor any agency thereof, nor any of their employees, makes any warranty, express or implied, or assumes any legal liability or responsibility for the accuracy, completeness, or usefulness of any information, apparatus, product, or process disclosed, or represents that its use would not infringe privately owned rights. Reference herein to any specific commercial product, process, or service by trade name, trademark, manufacturer, or otherwise does not necessarily constifute or imply its endorsement, recommendation, or ficvoritig by the United States Government or any agency thereof. The views and opinions of authors expressed herein do not necessarily state or ref'ect those of the United States Government or any agency thereof.
\end{abstract}

Prepared for

THE U.S. DEPARTMENT OF ENERGY AGREEMENT NO. DE-FG02-86ER 13552 


\section{Scope of Project for Total Funding Period (12/1/89-11/30/92).}

The objectives of this research, as stated in the proposal for the second term of funding, were to continue to further both the theoretical understanding of and the development of computer tools (algorithms) for separation processes involving nonideal mixtures. These objectives were divided into three interrelated major areas - the mathematical analysis of the number of steady-state solutions to multistage separation processes, the numerical analysis of general, related fixed-point methods, and the development and implementation of computer tools for process simulation.

\section{I.1. Mathematical Analysis}

The focus of this research was to build upon and refine our framework for analyzing the number of solutions to multistage separation processes under very mild conditions on phase equilibria and energy balance effects (i.e., only that the fluid phases satisfy the GibbsDuhem equation). This theoretical framework, originally developed by Sridhar and Lucia $(1989,1990)$, centered around the development of constructive analyses using a steady-state model, classical fixed-point theory, the implicit function theorem to build nonlinear maps from one specification set to another, and the analysis of the nonsingularity/singularity of the associated Jacobian matrices of these maps. Both binary and multicomponent mixtures were to be studied and the uniqueness or multiplicity of solutions was to be related to the characteristics of the mixture (i.e., homogeneous or heterogeneous) and/or the kinds of specific tions imposed on the separator. By homogeneous mixture, we mean a mixture that is stable with respect to phase splitting over the temperature, pressure and composition ranges of interest. A heterogeneous mixture, on the other hand, can potentially split into two phases for some conditions of temperature, pressure and composition.

Analysis during the first three year funding period (6/1/86-11/30/89) yielded successful proofs of the uniqueness of solutions for binary homogeneous multistage separators with specifications of fixed temperature and pressure (TP) profiles, fixed heat duty and pressure (QP) profiles and fixed reflux ratio and bottoms flow (RB). In all cases, the role of homogeneity in determining solution uniqueness was clearly identified. Proposed research for the second funding term included a completion of the analysis of binary homogeneous separators under a variety of product composition specifications. It was conjictured that these specification sets admit unique solutions because they uniquely fix the separation made by the column.

Successful analysis of the uniqueness of solutions for multistage separation processes with fixed temperature and pressure (TP) profiles ior multicomponent homogeneous mixtures was also produced during the first three year funding term. Again, the role of the homogeneity of the fluid phases in determining solution uniqueness was clearly shown. Further development of the analysis framework developed by Sridhar and Lucia was proposed for the second funding term and included both the analysis of the number of solutions to homogeneous multicomponent, multistage separators for other sets of specifications and the identifications of sources of and pathways to vapor-liquid solution multiplicity when conditions of homogeneity were relaxed. 


\section{I.2. Numerical Analysis}

Insights provided by mathematical analysis were conjectured to be useful in building a better understanding of the convergence behavior of existing algorithms and/or the development of new computer tools for steady-state process simulation. During the first and also as part of the second funding term, the mathematical analysis in Sridhar and Lucia (1989) was, in fact, used directly to understand the convergence characteristics of traditional sum-rates (SR) methods for separation process simulation and to develop a more reliable and efficient sum-rates method. Proposed research in this area was to continue along the same lines (i.e., using insights provided by mathematical analysis) and to include a study of the convergence characteristics of existing bubble point (BP) tearing methods as well as those of equation-solving methods such as direct substitution and Newton's method. In all cases, emphasis was to be placed the structure of basins of attraction, the presence of periodic/chaotic behavior and singular points, and the efiects of stabilization procedures like step bounding and trust region (dogleg) strategies on reliability and efficiency.

\section{I.3. Computer Tools}

The development of useful computer tools for the steady-state simulation of separation processes must, in our opinion, be done in a manner that is concomitant with mathematical and numerical analysis. Knowledge of the behavior of existing and/or new algorithms for simulation can only be gathered from reasonably reliable computer implementations of these methods and direct application on relevant examples. This, in turn, often provides other insights for further improvernent and/or new directions for development. Thus, numerical implementation, verification and testing of algorithms based on insights provided by both mathematical and numerical analyses on relevant separation process (sub)problems were proposed. It was conjectured that more reliable and efficient methods for simulation would result from this multilevel feedback procedure.

\section{Summary of Progress for Total Funding Period}

Research results for the total funding period include:

1. A complete and rigorous analysis of the number of solutions to binary homogeneous multistage separators under a variety of product flow and composition specifications;

2. A rigorous analysis of the number of steady-state solutions to homogeneous multicomponent, multistage separation processes for specifications of fixed heat duty and pressure profiles;

3. A numerical analysis of existing bubble point methods for narrow boiling separations and the development, analysis, implementation and numerical testing of a modified bubble point algorithm based on insights provided by the mathematical analysis in 1 and 2 ;

4. A numerical study and analysis of the convergence behavior of various fixed-point methods for separation process simulation in both the real and complex domains with 
emphasis on periodic/chaotic behavior, singular points and global convergence via stabilization.

5. The identification of one pathway to vapor-liquid solution multiplicity in the absence of homogeneity.

\section{II.1. Further Analysis of Binary Homogeneous Separators}

The objective of this study was to complete the mathematical analysis of the number of solutions to multistage separation processes involving binary homogeneous mixtures by considering product flow and composition specifications, which can be important in pollution abatement, profitability, safety and other process issues. Thus this study focused on theoretical developments using the implicit function theorem to map from specification sets for which uniqueness had been proved to product flow and/or composition specifications, analyzing the Jacobian matrices of these maps with regard to nonsingularity, and identifying the role of homogeneity, if any, in determining the number of solutions.

In our opinion, the important contributions of this work include the successful establishment of the uniqueness of steady-state solutions for a variety of product flow and/or composition specifications within the general mathematical analysis framework developed by Sridhar and Lucia (1989) and under very mild conditions on the phase equilibria. One important by-product of this analysis was both new and simpler proofs of some of the original analysis contained in Sridhar and Lucia (e.g., tighter bounds on and simpler expressions for the effects of changes in stage temperatures on molar flows in the column and a more accurate representation of the Jacobian matrix for the map from the set of TP specifications to the set of QP specifications). These analysis results were also related to Ponchon-Savarit diagrams for the purposes of both geometric illustration and accessibility at the undergraduate level.

These are both new and important results. In our opinion, they are important because they contribute to the literature on separation process analysis in a way that clearly identifies the role of homogeneity in determining the number of solutions and, at the same time, make the results of rigorous analysis accessible to the chemical engineering undergraduate.

\section{II.2. Multicomponent, Multistage Process Analysis}

The purpose of this research was to extend the analysis of binary homogeneous separators to multistage processes involving homogeneous multicomponent mixtures for specifications of fixed heat duty and pressure profiles. Again, the framework of Sridhar and Lucia was to be used, only mild conditions governing phase equilibria and energy balance effects were to be invoked, and the role of homogeneity in determining the number of solutions was to be carefully delineated.

Significant results in this area establish the uniqueness of steady-state solutions to multistage separation process models involving homogeneous multicomponent mixtures and specified heat duty and pressure profiles. Energy balances were used to construct a nonlinear map from the set of TP solutions (for which uniqueness had been proved) to the set of 
QP solutions using the implicit function theorem. The inverse nonnegativity of the second derivative matrix of the Gibbs free energy function projected onto the mass balance constraints, which only holds under conditions that the fluid phases are homogeneous, was then used to derive inequalities that establish the effect of changes in stage temperatures on molar flows throughout the separator. Using these results, it was shown that the Jacobian matrix for the map from the TP solutions to the QP solutions is nonsingular, thereby establishing that solutions to multistage separation processes involving homogeneous multicomponent mixtures and fixed heat duty and pressure profiles are unique.

In our opinion, these research results are very significant. Together with the results for the TP specification set, they represent the only successful rigorous analysis of multistage separation processes involving multicomponent mixtures. They also clearly identify the role of homogeneity in determining the number of steady-state solutions.

\section{II.3. Bubble Point Algorithms for Separation Processes}

The purpose of this research was to illustrate that our analysis framework provides constructive information that can be used to both understand the convergence behavior for existing algorithms and/or develop new methods for steady-state process simulation. Insights provided by the mathematical analyses of binary and multicomponent homogeneous separators were applied to bubble point methods for the simulation of the distillation of narrow boiling mixtures.

Significant results here include the development of a modified bubble point method based on insights provided by analysis, the numerical analysis of existing and modified bubble point methods, and the further demonstration that rigorous mathematical analysis can be constructive. Both the inner and outer loops of traditional bubble point methods were modified based on insights provided by the analysis. In particular, the inequalities derived from analysis that show the effects of changes in stage temperatures on molar flows in column were used to develop better Jacobian matrix approximations for the outer loop. Traditional and modified bubble point methods were compared using several literature examples. Numerical results showed that our modified bublle point method is more reliable and efficient than traditional bubble point methods. When both methods worked, the modified method usually required significantly less computer time than that required by traditional methods. Furthermore, the modified bubble point method was able to solve distillations involving wide boiling mixtures, whereas traditional methods were not. Finally, convergence and rates of convergence have been established and the practical value of rigorous analysis has been clearly demonstrated. A manuscript containing the details of these aspects of our research is under preparation.

In our opinion, these are important research results because they provide more reliable and efficient tearing algorithms for separation process simulation and because, together with the results of Sridhar and Lucia (1990) for modified sum-rates methods, they challenge the long-standing folklore associated with the numerical performance of equation-tearing methods (i.e., that SR and BP methods are only useful on wide- and narrow boiling mixtures respectively). 


\section{II.4. Convergence Behavior of Fixed-Point Methods}

The objective of this research was to study the convergence behavior of various common fixed-point methods used in separation process simulation. This included both pure methods, such as direct substitution and Newton method, as well as stabilization techniques such as step bounding and trust region (or dogleg) methods. Thus, the research in this area focused on the implementation, numerical analysis and testing of the aforementioned methods on a variety of heterogeneous separation process problems and subproblems.

Important contributions from this research include both the illustration that pure fixed-point methods can exhibit periodic and/or chaotic behavior on even simple separation process problems, the birth of the conceptual idea of performing process calculations in the complex domain and the development of a trust region method in the complex domain that appears to possess global convergence to a solution. Initial investigation of the convergence behavior of direct substitution and Newton's method in either an equation-tearing or equation-solving environment were conducted by Lucia et al. (1990). Small separation process examples (e.g., roots to equations of state, dewpoint and flash calculations and a simple two-stage separator) showed that both methods can exhibit periodic/chaotic behavior and that much of this nonconvergent behavior was accompanied by a bifurcation of a pair of real solutions into the complex domain. Clearly then, real initial values can not be used to find complex-valued solutions. These observations led to the controversial concepts of performing the calculations in the complex domain to improve reliability and complex-valued hypothetical thermodynamic states. This, in turn, led to a deeper geometric understanding of the associated Julia sets (i.e., set of points that are nonconvergent) and the observation that stabilization procedures such as traditional trust region methods restricted to the real domain can terminate at singular points (or saddlepoints in the least squares function). Finally, a trust region method in the complex domain based on singular point perturbation was developed. It was tested on a variety of separation process examples and was shown to provide global convergence to a solution. Details of these and other aspects of the stages of this numerical analysis can be found in the reprint of the paper by Lucia et al. (1990) contained in the Appendix and in preprints of manuscripts by Lucia and Taylor (1992) and Lucia et al. (1992), the latter two of which are certainly available on request.

In our opinion, these research results are important because they introduce a novel way of performing separation process calculations (i.e., in the complex domain) that avoids many computational difficulties (i.e., nondifferentiabilities across phase boundaries, near turning points and bifurcations, etc.) in a natural way and, at the same time, provide more reliable and efficient equation-solving methods for use in simulation.

\section{II.5. Pathways to Vapor-Liquid Multiplicity}

The objective of this research was to build an understanding of the sources of and pathways to vapor-liquid solution multiplicity for separation processes involving heterogeneous mixtures. There is a considerable amount of confusion and conflict in the literature in this regard that stems from the much-studied ethanol-benzene-water (EBW) azeotropic distillation and the fact that the solution multiplicity reported by several workers in this 
area occurs for specifications in which the liquid phases are stable on all stages in the column (see Magnussen et al, 1979; Kovach and Seider, 1987; Venkataraman and Lucia, 1988). This is contrasted with the reports of vapor-liquid multiplicity at the single-stage level in which only one solution normally has a stable liquid (see Van Dongen et al., 1983). Thus this study focused on constructing a simple distillation involving a heterogeneous mixture that captured all of the essential attributes of the EBW distillation in an effort to uncover the sources of solution multiplicity.

Significant contributions of this research include the simple two-stage distillation of acetonitrile, water and acrylonitrile, as presented in Lucia et al. (1990), that exhibits vaporliquid solution multiplicity and a parametric study in molar vapor boilup and distillate rate that shows that solution multiplicity in a multistage process can occur because of multiplicity at the single-stage level and that this, in turn, is a consequence of the heterogeneous nature of the liquid phase. The initial construction of a simple distillation with all of the essential attributes of the EBW distillation is due to Lucia et al. (1990), who used this problem to illustrate that simple process models could exhibit chaos. However, we believed this problem could also be used to study vapor-liquid solution multiplicity in a way that perhaps could reveal the source of multiplicity. An initial parameterization in both vapor boilup and distillate rate was conducted and multiple vapor-liquid solutions with stable liquid phase throughout the column were found. Continued parameterization by lowering the vapor boilup, while keeping the distillate to boilup ratio relatively high, showed that these vapor-liquid solutions all stem from vapor-liquid solution multiplicity at the single-stage level. Furthermore, the transition from multiplicity at the single-stage level to multiplicity at the multistage level proceeds from a lariat-shaped solution curve to the more familiar S-shaped curve, like the one shown in Fig. 15 in Lucia et al. (1990), and can move in anci out of the homogeneous regime due to the nonlinear interaction between the stages. The details of this study are contained in a draft of a manuscript by Lucia and Guo and will soon be submitted for publication.

In our opinion, these research results are important because they present the first satisfactory explanation of at least one source of and pathway to vapor-liquid solution multiplicity in multistage separations involving heterogeneous mixtures. In addition, this work complements and extends prior work in this area in a way that partly brings together the vast amount of fragmented knowledge that exists with regard to the separation of heterogeneous mixtures.

\section{Proposed Research Plans for the Remainder of Funding Period}

Our proposed research plans for the remainder of the funding period are to continue the development of analysis and computer tools for multistage separation processes. In particular, we plan to

1. develop the analysis of the number of solutions to binary homogeneous azeotropic separation processes under a variety of specification sets,

2. continue to develop the analysis of the number of solutions to homogeneous multi- 
component, multistage separation processes for various specifications,

3. continue to study issues in vapor-liquid solution multiplicity in the absence of homogeneous, and

4. continue the studies in separation process simulation in the complex domain.

This proposed research will, in fact, carry us beyond the end of the funding period. Consequently, it also constitutes part of a renewal proposal for further funding of research in analysis and algorithms for multistage separation processes.

\section{Compliance}

Comparison of the proposal for this funding term and this report shows that the research work to date and that planned for the remainder of the total funding period are in compliance with the original agreement on which the grant was awarded.

\section{Personnel}

The current grant is under the direction of Dr. Angelo Lucia, Professor of Chemical Engineering at Clarkson University. The approximate time commitment of the principal investigator has been one (1) month during summers $(1990,1991,1992)$ and approximately four (4) months during the academic years for the current funding period. The duties of the PI have been the normal administrative, technical support and technical development duties and are expected to continue with the same relative time commitment for the remainder of the funding period, as well as future periods of funding.

This grant also has been and/or is currently being used to support the following students:

1. Mr. Lakshmi Sridhar, duration of support: 9/1/86-4/1/91, completed Ph.D. degree $4 / 8 / 91$.

2. Mr. Xinzhou Guo, duration of support: 9/1/88-present, completed M.S. degree 8/90, currently Ph.D. candidate.

3. Mr. Hailang Li, duration of support: 9/1/90-present, Ph.D. candidate.

Dr. Sridhar completed his Ph.D. degree requirements in April, 1991. Approximately one and a half years of funding from this present term were used to support him during which time he developed a numerical analysis and performed numerical tests on both existing and new bubble point methods for separation process simulation. This work was done by him as a continuation of his responsibilities for all research aspects associated with separation processes involving homogeneous mixtures. Mr. Guo, on the other hand, is responsible for all research aspects associated with separation processes involving heterogeneous mixtures, completed his M.S. thesis in August, 1990 and has progressed to the Ph.D. program. Finally, 
Mr. Li has assumed the responsibility of all remaining and any future aspects of research associated with separation processes involving homogeneous mixtures.

\section{Publications and Presentations}

\section{Publications}

1. L.N. Sridhar and A. Lucia, "Analysis of Multicomponent, Multistage Separation Processes: Fixed Temperature and Pressure Profiles", Ind.\& Eng. Chem. Res., 29, 1668 (1990).

2. L.N. Sridhar and A. Lucia, "Tearing Algorithms for Separation Process Simulation", Comput. Chem. Engng., 14, 901 (1990).

3. A. Lucia, X. Guo, P.J. Richey and R. Derebail, "Simple Process Equations, FixedPoint Methods and Chaos", AIChE J., 36, 641 (1990).

\section{Submitted for publication during total funding period}

1. A. Lucia and H. Li, "Constrained Separations and the Analysis of Binary Homogeneous Separators", Ind.\&Eng. Chem. Res. (1992).

2. A. Lucia, X. Guo and X. Wang, "Process Simulation in the Complex Domain", AIChE $J .(1992)$

\section{Manuscripts in preparation}

1. A. Lucia and H. Li, "Multicomponent, Multistage Separation Process Analysis".

2. A. Lucia, L. N. Sridhar and H. Li, "An Analysis of Bubble Point Methods"

3. A. Lucia, "Pathways to Vapor-Liquid Solution Multiplicity".

\section{Presentations}

1. L.N. Sridhar and A. Lucia, "Analysis of Multicomponent, Multistage Separation Processes", AIChE Annual Meeting, San Francisco, CA, November 1989.

2. A. Lucia, X. Guo, P.J. Richey and R. Derebail, "Simple Process Equations, FixedPoint Methods and Chaos", AIChE Annual Meeting San Francisco, CA, November 1989.

3. A. Lucia and X. Guo, "Chaotic Process Simulation", AIChE Spring National Meeting, Orlando, FL, March 1990. 
4. A. Lucia, "Chaotic Behavior of Fixed-Point Methods", Can.Chem.Eng.Conf., Halifax, $N S$, July 1990.

5. A. Lucia and X. Guo, "Chaotic Behavior of Newłon's Method in the Complex Domain", AIChE Annual Meeting, Chicago, IL, November 1990.

6. A. Lucia, X. Guo and X. Wang, "Process Simulation in the Complex Domain", AIChE Spring National Meeting, New Orleans, LA, March 1992.

7. A. Lucia and R. Taylor, "Complex Iterative Solutions to Process Model Equations", European Symposium on Computer Aided Process Engineering (ESCAPE-1), Elsinore, Denmark, May 1992.

\section{References}

1. Kovach, J. W. and W. D. Seider, "Heterogeneous Azeotropic Distillation HomotopyContinuation Methods", Comput. Chem. Eng., 11, 593 (1987).

2. Magnussen, T., M. L. Michelsen and A. Fredunslund, "Azeotropic Distillation Using UNIFAC", Third International Symposium on Distillation, Institute of Chemical Engineers Symposium Series 56; ICE: Rugby, England, 4.2, 1 (1979).

3. Sridhar, L. N. and A. Lucia, "Analysis and Algorithms for Multistage Separation Processes". Ind. Eng. Chem. Res., 28, 793 (1989).

4. Van Dongen, D. B., M. F. Doherty, and J. R. Haight, "Material Stability of Multicomponent Mixtures and the Multiplicity of Solutions to Phase Equilibrium Equations. 1: Nonreacting Mixture," I \& EC Fund, 22, 472 (1983).

5. Venkataraman, S. and A. Lucia, "Solving Distillation Problems by Newton-like Methods", Comput. Chem. Eng., 12, 55 (1988). 
Appendix

Reprints of Papers, Thesis Abstracts

Rernowal ard cycad separately-

II 
$\therefore$
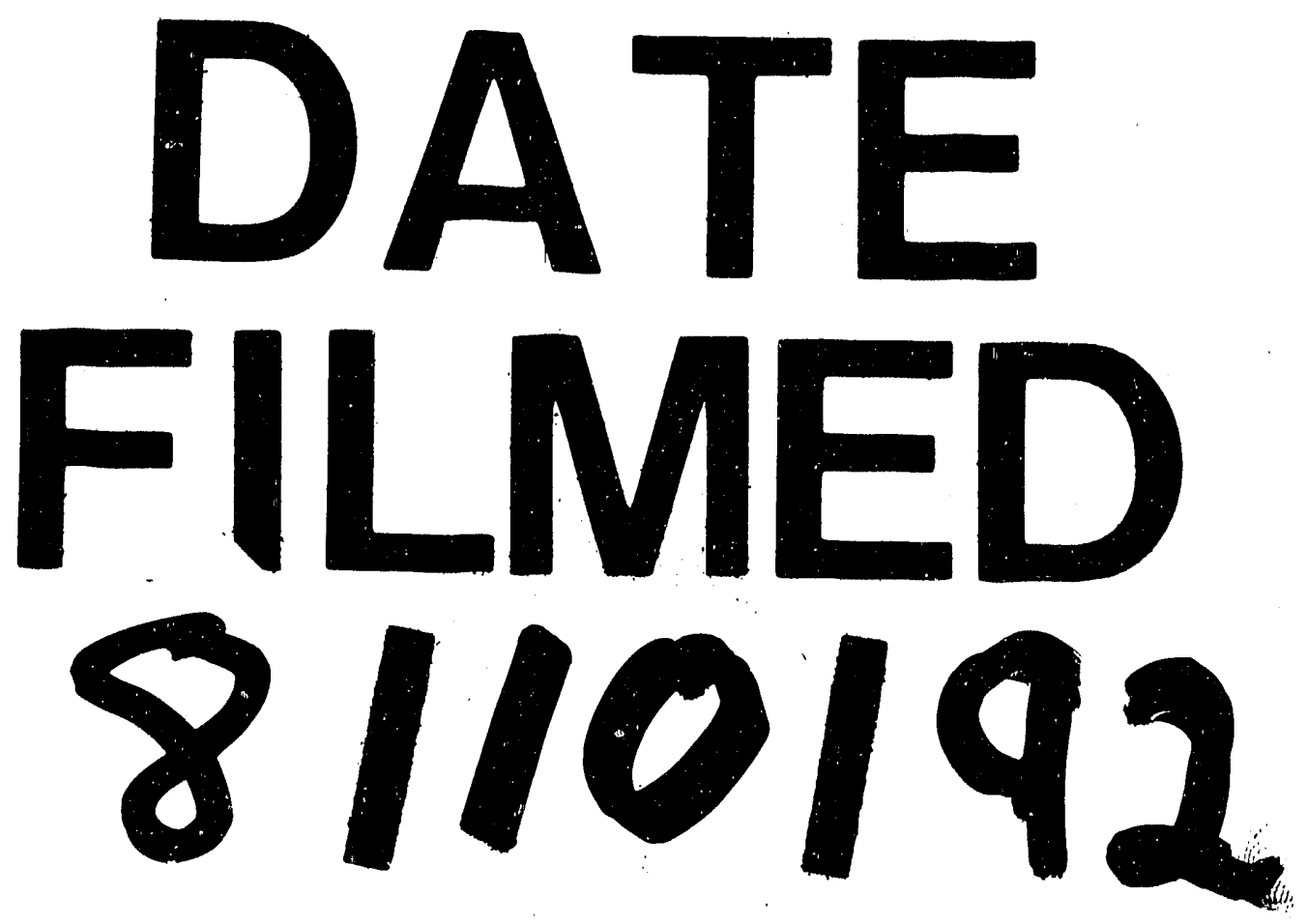